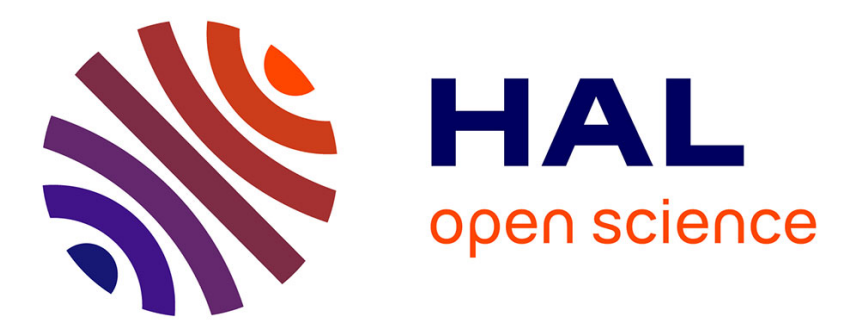

\title{
Nanometric sensing with laser feedback interferometry
}

Daeyoung Choi, Michael J Wishon, E. A Viktorov, D. S Citrin, A. Locquet

\section{To cite this version:}

Daeyoung Choi, Michael J Wishon, E. A Viktorov, D. S Citrin, A. Locquet. Nanometric sensing with laser feedback interferometry. Optics Letters, 2019, 44 (4), pp.903. 10.1364/ao.XX.XXXXXX . hal-03006991

\section{HAL Id: hal-03006991 \\ https://hal.science/hal-03006991}

Submitted on 16 Nov 2020

HAL is a multi-disciplinary open access archive for the deposit and dissemination of scientific research documents, whether they are published or not. The documents may come from teaching and research institutions in France or abroad, or from public or private research centers.
L'archive ouverte pluridisciplinaire HAL, est destinée au dépôt et à la diffusion de documents scientifiques de niveau recherche, publiés ou non, émanant des établissements d'enseignement et de recherche français ou étrangers, des laboratoires publics ou privés. 


\title{
Nanometric sensing with laser feedback interferometry
}

\author{
Daeyoung Chol ${ }^{1}$, Michael J. Wishon ${ }^{1}$, E. A. Viktorov², D. S. Citrin ${ }^{1}$, And A. Locquet ${ }^{1}{ }^{*}$ \\ ${ }^{1}$ Georgia Tech-CNRS UMI 2958, Georgia Tech Lorraine, 2 Rue Marconi 57070, Metz, France and School of Electrical and Computer Engineering, Georgia \\ Institute of Technology, Atlanta, Georgia 30332-0250 \\ 2 ITMO University, 14 Birzhevaya liniya, Saint Petersburg 199034, Russia \\ *Corresponding author: alocquet@georgiatech-metz.fr \\ Compiled November 16, 2020
}

\begin{abstract}
We demonstrate a nanometric sensor based on feedback interferometry in a DFB laser by using a measurement of either the optical frequency or laser voltage. We find that in an optimal range of optical feedback, the sensor operates reliably down to an extrapolated $12 \mathrm{~nm}$; for the sensor demonstrated here at $\sim 1550 \mathrm{~nm}$, this provides a minimum detectible displacement of $\lambda / 130$. ๑ 2020 Optical Society of America
\end{abstract}

OCIS codes: (140.3490) Lasers, distributed feedback; (140.5960) Semiconductor lasers; (280.0280) Remote sensing and sensors

http://dx.doi.org/10.1364/ao.XX.XXXXXX

Nanodisplacement sensors have been demonstrated based on a variety of physical effects and for a range of applications [1,2]. The laser feedback interferometry (LFI) or self-mixing interferometry sensor [3,4] we demonstrate is a noncontact sensor, and therefore we set aside discussion of most MEMS-based approaches. As for noncontact nanodisplacement sensors, there are a number of approaches, including those based on capacitance, eddy currents, and atomic-force microscopy [5,6]. These approaches, while in some cases sensitive to atomic-scale displacements can be complex, costly, and sensitive to quite small displacement ranges (small dynamic range).

LFI is based on light, reflected back into the active region, which in turn affects the laser diode (LD) operation in a fashion dependent on the phase of the reflected light $[7,8]$. LFI itself provides sensitivity at least on the scale of the emission wavelength $\lambda$, though below we show that the minimum detectible displacement (MDD) can be $\ll \lambda$.

A LD with optical feedback is generally known as an external-cavity semiconductor laser (ECL). Heuristically, when the target distance $L$ is varied by $\Delta L$, the properties of an ECL (frequency $v$, linewidth, and threshold gain) undergo periodic variation, which is broadly understood on the basis of the Lang and Kobayashi (LK) equations [9]. The feedback-phase sensitivity of the LFI sensor depends on the change of frequency $v$ with $L$, which in turn is also manifested in the optical output power $P_{\mathrm{PD}}$ measured by a photodiode (PD) and change of carrier concentration measured by the LD terminal voltage $V_{\mathrm{LD}}$ (under constant injection current $J$ ).

We demonstrate LFI sensors enabling unprecedentedly high sensitivity providing a linear sawtooth dependence on $L$ of the sensing parameter, here focusing on $V_{\mathrm{LD}}$.

LFI has been exploited for imaging [10, 11], biomedical sensing [12] and measuring absolute distance [13, 14], displacement [15-17], reflective index [18], velocity [19, 20], and flow [21]. Recently, Keeley et al. [22] investigated the emission spectrum of a THz QCL by measuring $v$ and $V_{\mathrm{LD}}$ as functions of the displacement $\Delta L$ and showed that LFI can achieve a similar spectral resolution as Fouriertransform infrared spectroscopy.

LFI displacement sensors have been demonstrated usually using a moving target, e.g. a harmonic piezo-actuator or loudspeaker that sweeps several $\mu \mathrm{m}$, combined with high sampling rates to measure fringes of the LFI signals. Their MDDs are from a few to hundreds of nm but require post-processing of the LFI signals and/or substantial modifications of the basic LFI setup [23-28], laser feedback grating $(<10 \mathrm{~nm})$ [26], Fourier transform $(\lambda / 50)$ [27], phase shifting $(\lambda / 12)$ [28-30]. In contrast, our approach requires neither modification of the basic LFI setup nor any signal processing, but relies on the direct physical measurement of the LFI signals in parameter ranges leading to high linearity and signal-to-noise ratio (SNR). Specifically, we demonstrate a LFI sensor with a MDD of $\lambda / 130$, assuming the feedback level can be adjusted in an optimal range. Since the LFI sensing mechanism is based on the feedback phase, such a sensor can also be used as a gas or liquid sensor using refractive index change. For the MDD and geometry we focus upon, this translates into the ability to sense refractive-index changes in the optical feedback loop of $\Delta n_{\text {min }} \sim 10^{-8}$.

In this Letter, because of the key rôle played by $v$ in LFI, we start with its dependence on $L$ before proceeding to focus on $V_{\mathrm{LD}}$. We first review important theoretical points that describe the variation in $v$ and consequently in $V_{\mathrm{LD}}$ with $L$.The theory of LFI is discussed in Refs. [7,31]. Briefly, the excess-phase equation derived from the LK model relates $\Delta L$ to $\Delta v=v-v_{0}$ as a periodic function where $v$ is the output frequency of the ECL and $v_{0}$ is the frequency of the free-running LD (i.e., in the absence of optical feedback). When $\Delta L$ changes, $\Delta v$ obeys $\Delta v(\Delta L)=m F(\Delta L)$ with $m$ the optical-feedback modulation index that defines the signal slope with respect to $\Delta L, F(\Delta L)$ a periodic function with period $\lambda_{0}$ where $\lambda_{0}=c / v_{0}$ with $c$ the speed of light in vacuo. Qualitatively, the dependence of $v$ on $\Delta L$ tends to be sinusoidal for weak feedback. The shape can become close to a piecewise linear function, 


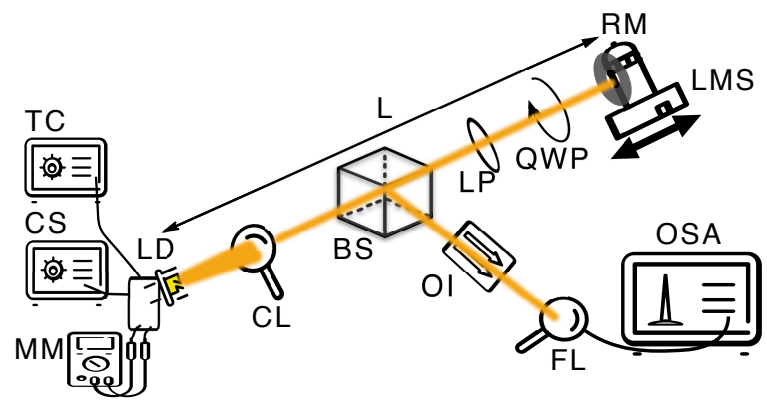

Fig. 1. Experimental setup. LD: laser diode, L: external-cavity length, TC: temperature controller, CS: current supply, CL: collimation lens, BS: beam splitter, LP: linear polarizer, QWP: quarter-wave plate, RM: reflecting mirror, LMS: linear motor stage, OI: optical isolator, MM: multimeter, FL: focusing lens and OSA: optical spectrum analyzer.

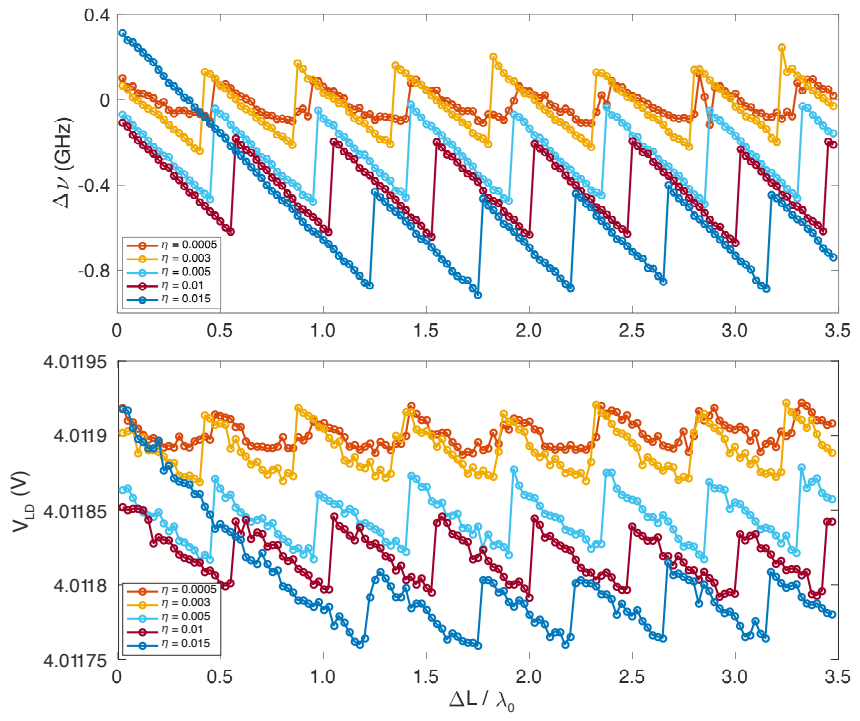

Fig. 2. $\Delta v$ (top) and $V_{\mathrm{LD}}$ (bottom) versus $\Delta L$ (in units of $\lambda_{0}$ ) from experiment. $\Delta L$ is characterized by movement away from the LD $(40 \mathrm{~nm} / \mathrm{step}) . L=30 \mathrm{~cm}, J=69 \mathrm{~mA}$, and $v_{0}=193281.8 \mathrm{GHz}$. The signal gradually becomes a piecewise linear function known as a regular sawtooth for $0.003<\eta<0.01$, corresponding conventionally to moderate feedback level $(1<C<4.6)$ ). In the curve for $\eta=0.015$, it is visible that some fringes are skipped, which occurs in the strong feedback regime $(C>4.6)$.

also known as a sawtooth wave, at moderate feedback level with discontinuities due to mode hopping to a neighboring externalcavity mode (ECM) $[3,4]$ with period $\lambda / 2$. Also, increasing feedback level results in greater amplitudes of $\Delta v$ over $\Delta L$. To conclude the review of the excess-phase equation, we mention that the $C$ parameter, defined e.g. in [4], is conventionally used to qualitatively classify the regimes from various self-mixing interferometry systems [4].

A schematic diagram is shown in Fig. 1. We use a single-longitudinal-mode multi-quantum well InGaAsP DFB laser [32]. The LD emits at $\lambda_{0} \sim 1550 \mathrm{~nm}$ with a free-running threshold current $J_{t h}=29.8 \mathrm{~mA}$. The LD is unpackaged providing direct access to the LD injection terminals. $J$ and temperature are stablized to an accuracy of $0.01 \mathrm{~mA}$ and $0.01 \mathrm{~K}$. The light is split via a $50 / 50$ beam splitter. The distance between the reflecting mirror (RM) and the LD determines $L$. RM is not diffusive, and the quarter-wave plate (QWP) is used to control the feedback with high precision. A reflection cancellation technique [33] can be applied for the measurements from diffuse surfaces. One optical path is adjusted for experimental feedback strength $\eta$ and $L$. The other is used to observe the optical spectrum (10 MHz resolution, Aragon Photonics BOSA 200C). The RM is placed on a linear motor stage (LMS, Newport XMS50) whose position is adjusted by a driver (Newport XPS-RL2) with $40 \mathrm{~nm} / \mathrm{step}$, error $< \pm 10 \mathrm{~nm}$; this error limits our ability to determine the MDD. A motorized piezo-actuation stage adjusts the angle of QWP with $0.01^{\circ}$ precision relative to the polarizer such that we can change $\eta$; conventionally, the maximum $\eta=1$ occurs when $\sim 18 \%$ of the optical power is fed back onto the emitting facet of the LD. $V_{\mathrm{LD}}$ is directly measured by a multimeter (Agilent 34410A) with $51 / 2$ digit-resolution and $0.003 \%$ accuracy.

We present our experimental results pertaining to nano-displacement sensing. $\Delta v$ is related to small variations $\Delta L$ based on the excess phase equation as discussed above. Working in the vicinity of $L=30 \mathrm{~cm}$, we adjust $\Delta v$ by changing $L$ in $40 \mathrm{~nm}$ steps using the LMS. Figure 2 shows $\Delta v$ and $V_{\mathrm{LD}}$ versus $\Delta L$ beginning at very weak $\eta$. For $\eta=0.0005, \Delta v$ exhibits a noisy skewed sine-like behavior, corresponding conventionally to the weak feedback regime $(C<1)$. As $\eta$ is increased, the dominant optical peak at $v$ is shifted down in frequency with respect to $v_{0}$ and displays increasingly pronounced sawtooth behavior with discontinuity periods $\lambda / 2$. When the linewidth of a single longitudinal mode extends over the external-cavity free-spectral range $f_{\mathrm{ECM}} \simeq 460 \mathrm{MHz}$, at very weak $\eta$ (i.e., 0.0005 in Fig. 2), this leads to a smooth transition to a neighboring ECM. As $\eta$ is increased, the linewidth of ECL is narrowed (« 40 


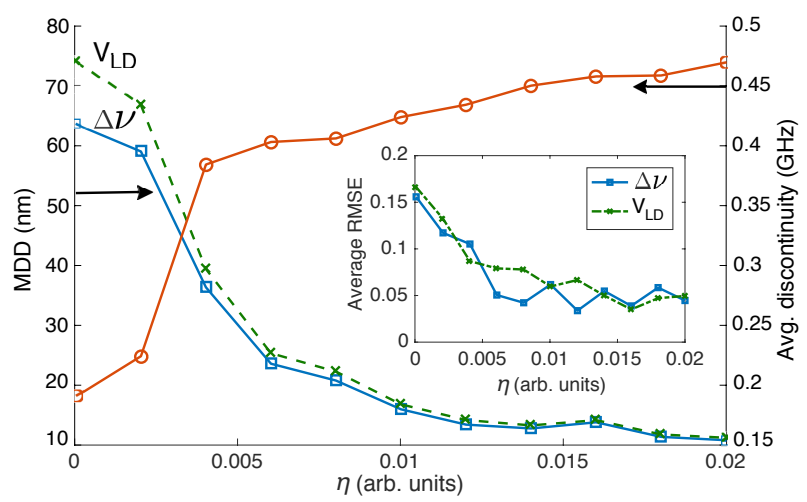

Fig. 3. MDD determined by $\Delta v$ and $V_{\mathrm{LD}}$ and average discontinuity in $\Delta v$ as a function of $\eta$. The average RMSE (inset) displays the deviation of the differences between the best linear fit and measured values. Lower RMSE for $\eta>0.01$ leads to MDD=12 nm.

$\mathrm{MHz}$ ), thereby inducing abrupt discontinuities. When $\eta \geq 0.01$ (Fig. 2), the initial sawtooth periods for small $\Delta L$ are lost. This is because the single-mode laser dwells on one of several ECMs prior to jumping to another ECM [34]. Since the results in Fig. 2 are obtained by moving the target away from the LD, the slopes of the linear variations in $\Delta v$ are negative. The slopes are positive when the target moves closer to the LD and hysteresis is observed (not shown). Sine-like LFI signals correspond to weak feedback, sawtooth to moderate, and loss of sawtooth periods to strong feedback [4, 35].

As expected from the theory, we observe sawtooth behavior in $\Delta v$ as well as $V_{\mathrm{LD}}$ with periodic discontinuities separated by $\lambda / 2$ in $\Delta L$. Of note, the sawtooth behavior in $\Delta v$ is not around 0 (i.e., the value in the absence of feedback) because $v$ is shifted as soon as $\eta$ is applied. Larger $\eta$ leads $v$ to be shifted further away from $v_{0}$ [36]. Sawtooth behavior appears as long as the ECL shows continuous-wave (CW) operation. The ECL can be destabilized by complicated dynamics with undamped relaxation oscillations [37] at which point sawtooth behavior is suppressed.

It is useful to trace the impact of the experimental feedback strength $\eta$ on ECM spectra. We make this analysis via the side-mode suppression ratio (SMSR), the ratio between the dominant mode and the largest neighboring ECM, which is typically $f_{\mathrm{ECM}}$ away in the optical spectrum [38]. We can thus track the transition from single-mode behavior to one where several ECMs are active around $v$ by considering the SMSR. The participation of more than one ECM will lead to more complicated dynamics, and consequently degrade LFI signals. We increase $\eta$ from the weakest possible value and monitor the crossover from weak to moderate feedback. For weak feedback, the ECL emits on only one ECM, corresponding to CW operation, and SMSR $\gtrsim 35 \mathrm{~dB}$. For moderate feedback, the ECL still operates on one ECM for a given $L$, but the SMSR is slightly degraded ( $25 \mathrm{~dB}<\mathrm{SMSR}<35 \mathrm{~dB}$ ), revealing the participation of several ECMs. As $\eta$ is further increased, the number of ECMs increases and some periods of the sawtooth are lost [4]. SMSR appears to be a useful tool to determine the feedback range where sawtooth periods are observed.

We have concentrated attention on the behavior of $\Delta v$ as $L$ is varied. $v$ and the carrier density are related since the effective refractive index and the threshold gain vary with optical feedback [16]. In turn, the variation of carrier density is manifested in $V_{\mathrm{LD}}$ [39], providing a more practical LMI signal than $\Delta v$.

We now optimize MDD. Our focus is on $\Delta v$; since $\Delta v$ is highly correlated with $V_{\mathrm{LD}}$, optimal feedback conditions will also apply well to $V_{\mathrm{LD}}$ as the LFI signal. We measure the magnitude of the discontinuities in $\Delta v$ for various $\eta$. As shown in Fig. 3, increasing $\eta$, the average discontinuity is increased, and then stays around at $f_{\mathrm{ECM}} \simeq 460 \mathrm{MHz}$ for which we observe clear sawtooth periods in $\Delta v$. It is also well-known that as an ECM is born through a feedback increase [3, 4], the distance from its closest neighbor increases with feedback strength until it reaches a value close to $f_{\mathrm{ECM}}$.

Let $G$ be the LFI signal measured (e.g., $\Delta v, \Delta P_{\mathrm{PD}}$ (change in photodiode output power), or $\Delta V_{\mathrm{LD}}$ (change in the LD terminal voltage for the given $J$ with respect to the case of $\eta=0$ ). The ideal sensitivity is $d G / d L=s$ where $s$ is the signal slope with respect to $L$ (neglecting discontinuities in $G$ ). It is therefore advantageous to choose parameters to maximize $|s|$. Due, however, to unavoidable sensitivity error, $\operatorname{MDD}\left(\Delta L_{\text {min }}\right)$ also depends on the error variations $n(L)$ in $G$. To find $\Delta L_{\text {min }}$, we begin with $G$ including $n(L)$, $G=s L+n(L) . \Delta L$ will produce a change $\Delta G$ in $F$ given by

$$
\Delta G=\frac{d G}{d L} \Delta L \approx s \Delta L+[n(L+\Delta L)-n(L) \cdot]
$$

This can be detected provided $\Delta L \gg \frac{1}{s}[n(L+\Delta L)-n(L)]$. This quantity on average is independent of $L$ though it does depend on $\Delta L$. We can simply identify the quantity in brackets on the right-hand side of the equation as a characteristic noise amplitude $n_{\text {avg }}$. Namely, suppose it is adequate to identify $n_{\text {avg }}$ with the root-mean square error (RMSE) between experimental G and its best linear fit. We therefore approximate values for the MDD as $\Delta L_{\min }=\frac{1}{s} n_{\text {avg. }}$. This analysis is expected to be valid so long as the correlation length of $n(L)$ is less than the MDD. We find that when the discontinuity in $v$ is locked at $\sim f_{\mathrm{ECM}}$, the linewidth of the $v$ gets narrower and highly linear behavior of $\Delta v$ between the discontinuities is observed, resulting in significantly reduced RMSE of $v$ (inset of Fig. 3). As shown in Fig. $3(0.015<\eta<0.02)$, MDD obtained by $v$ is as small as $12 \mathrm{~nm}$, which is very close to the experimentally limited resolution $(10 \mathrm{~nm})$ of the LMS, strongly suggesting that the LMS is itself a major limitation. Let us note that, some sensing applications require measurements in the longer displacement range and may benefit from a recently suggested 


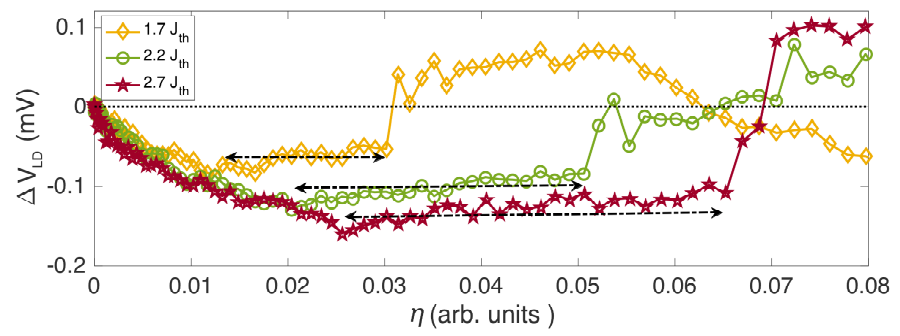

Fig. 4. $\Delta V_{\mathrm{LD}}$ as a function of $\eta$. A certain range of $\Delta V_{\mathrm{LD}}<0$ is observed before jumping back to 0 for larger values of $\eta$. The double arrowed lines indicate the optimal $\eta$ range for improved MDD. Higher $J$ leads to longer intervals of $\eta$ where $\Delta V_{\mathrm{LD}}<0$.

feedback phase retrieval algorithm, called Consecutive Samples based Unwrapping [40]. However, our focus in this work is to directly measure a minimum detectible displacement.

We have verified that $\Delta v$ and $\Delta V_{\mathrm{LD}}$ are highly correlated by programming the LMS to change its position with random step size in the range $40-500 \mathrm{~nm}$. We find the correlation coefficient reaches $\sim 0.96$ when the discontinuity saturates at $\sim f_{\mathrm{ECM}}$, i.e., when $0.01<\eta<0.02$ in Fig. 3. When no clear sawtooth in $\Delta v$ is observed at very weak $\eta$ (i.e., $\eta<0.0005$ in Fig. 3), the correlation coefficient between $\Delta v$ and $\Delta V_{\mathrm{LD}}$ is reduced to $\sim 0.88$. The lower correlation at weaker $\eta$, we suspect, is due to the broad linewidth of the ECL, which leads $v$ and $V_{\mathrm{LD}}$ to be susceptible to noise.

To verify MDD based on $V_{\mathrm{LD}}$, as an example at $L=30 \mathrm{~cm}$, when $\Delta v$ and $\Delta V_{\mathrm{LD}}$ vary with $\Delta L$, the slope $d G / d L\left(i . e ., d v / d L, d V_{\mathrm{LD}} / d L\right)$ gives values of $1.2 \mathrm{MHz} / \mathrm{nm}$ and $0.25 \mu \mathrm{V} / \mathrm{nm}$, which can be converted to $d V_{\mathrm{LD}} / d v \approx 0.2 \mu \mathrm{V} / \mathrm{MHz}$. With RMSE computed at various $\eta$ for $\Delta L_{\text {min }}$, we can derive MDD by $\Delta V_{\mathrm{LD}}$, enhanced close to MDD based on $v$ when the discontinuity is $\sim f_{\mathrm{ECM}}$. This observation indicates that $\Delta V_{\mathrm{LD}}$ can be used to detect displacement on a par with $\Delta v$ at a certain range of $\eta$.

As the next step, we find the optimal range of $\eta$ minimizing MDD by $\Delta V_{\mathrm{LD}}$. As shown in Fig. 4 , when $\eta$ is increased, $\Delta V_{\mathrm{LD}}$ typically decreases, plateaus, then increases. This negatively shifted plateau corresponds to where the discontinuity sticks at $\sim f_{\mathrm{ECM}}$, for nano-displacement sensing. In addition, we can see that the plateau tends to broaden with increasing $J$ because stronger $\eta$ is required to render an ECL unstable [41]. Beyond the plateau, SMSR is significantly degraded to less than $25 \mathrm{~dB}$, indicating that more than two ECMs are involved in the ECL dynamics. Thus, it is difficult to maintain a periodic sawtooth. Of note, the procedure for determining the optimal $\eta$ range does not require any movement of the target.

Optimal $L$ is now considered. Assuming $\Delta L$ will be at most a few $\lambda$, what is critical is that $f_{\mathrm{ECM}}$ does not become so small that more than one ECM contributes to the emission at $\eta$ required to keep the discontinuity of $v$ at $\sim f_{\mathrm{ECM}}$. Once more than one ECM begins to participate, the ECL becomes more susceptible to noise and begins to exhibit complex dynamics related to undamped relaxation oscillations. To understand how this affects sensor performance, we vary $L$ by $10 \mathrm{~cm}$ increments and find that when $L \geq 60 \mathrm{~cm}$, the ECL tends to lose stability for LFI even with a very low $\eta$ resulting in SMSR $<25 \mathrm{~dB}$ and frequent mode hopping. Thus, combined with the observation that larger $J$ enables sawtooth behavior for a broader range of $\eta$, sawtooth behavior in $\Delta v$ and $\Delta V_{\mathrm{LD}}$ can be maintained when $J \gtrsim 1.5 J_{t h}$ and $L<60 \mathrm{~cm}$; however, the details will depend on $\lambda$ and other LD parameters.

To check the robustness of the results, we conducted experiments using an ECL based on a packaged DFB laser to monitor $\Delta P_{\mathrm{PD}}$ using the internal PD. The LFI signals were found to have no significant difference between $\Delta P_{\mathrm{PD}}$ and $\Delta V_{\mathrm{LD}}$. The previous observations are dependent on the characteristics of our multimeter, whose noise floor likely limits the accuracy of the measurement. Other authors have concluded that signals obtained by internal PDs have slightly better SNR than those from $\Delta V_{\mathrm{LD}}[16,42,43]$; however, we did not observe the negative shifted behavior in $P_{\mathrm{PD}}$ with increasing $\eta$ unlike $\Delta V_{\mathrm{LD}}$ as shown in Fig. 4 . Under conditions where $\Delta P_{\mathrm{PD}}$ and $\Delta V_{\mathrm{LD}}$ are affected by independent noise sources, $\Delta V_{\mathrm{LD}}$ can be used to calibrate optimal $\eta$ when it is negative. Thus, it seems reasonable to use $\Delta V_{\mathrm{LD}}$ when an internal PD is not available because $\Delta V_{\mathrm{LD}}$ provides a direct measurement and eliminates noise due to optical-to-electrical conversion for the PD. Additionally, $P_{\mathrm{PD}}$ can be affected by stray light [39].

As the physical basis of LFI sensing is the sensitivity of the ECL operation to changes in optical phase, the change of refractive index within the external cavity due to a transparent gas or a liquid can be sensed with the LFI technique. To translate the results to this domain, suppose we wish to determine the minimum detectible refractive index change (MDRIC) $\Delta n_{\text {min }}$ associated with the minimum relevant feedback-phase change $\Delta \phi_{\min }$. We have $\Delta \phi_{\min }=4 \pi L \Delta n_{\min } / \lambda$ or $\Delta n_{\min }=\frac{\lambda \Delta \phi_{\min }}{4 \pi L}$. For conventional interferometry, $\Delta \phi_{\min } \sim \pi / 2$, and so for our parameters $(L=30 \mathrm{~cm}, \lambda=1550 \mathrm{~nm}), \Delta n_{\min }=6.5 \times 10^{-7}$. For LFI, however, $\Delta \phi_{\min }$ is determined by the MDD as $\Delta \phi_{\min }=\frac{4 \pi(\mathrm{MDD})}{\lambda}$, which for our parameters is $4 \pi / 130$. This gives $\Delta n_{\min }=4.0 \times 10^{-8}$, a 16-times improvement over the sensitivity of conventional interferometry. This is also to be compared with the performance observed in Ref. [18] where MDRIC of $\Delta n_{\min }=2.0 \times 10^{-5}$ was reported.

To conclude, we have experimentally investigated an LFI sensor based on an ECL. By selecting the operating regime with highest correlation and linearity between $\Delta v$ and $\Delta V_{\mathrm{LD}}$, we obtain an extrapolated MDD of $12 \mathrm{~nm}$ or $\lambda / 130$, a record for a sensor making a direct use of the native LFI signal. MMD of $12 \mathrm{~nm}$ is likely due to limitations of our piezo-actuator with a precision of $10 \mathrm{~nm}$ and the multimeter resolution used to make $V_{\mathrm{LD}}$ measurements.

The authors thank Conseil Régional du Grand Est for financial support and Prof. Julien Perchoux for insightful advice.

\section{REFERENCES}

1. H. Hansen, K. Carneiro, H. Haitjema, and L. D. Chiffre, CIRP Annals 55, 721 (2006). 
2. A. J. Fleming, Sens. Actuator A-Phys. 190, 106 (2013).

3. S. Donati, Laser Photonics Rev. 6, 393 (2012).

4. T. Taimre, M. Nikolić, K. Bertling, Y. L. Lim, T. Bosch, and A. D. Rakić, Adv. Opt. Photon. 7, 570 (2015).

5. D. J. Bell, T. J. Lu, N. A. Fleck, and S. M. Spearing, J. Micromechanics Microengineering 15, S153 (2005).

6. M. A. Haque and M. T. A. Saif, Exp. Mech 43, 248 (2003).

7. K. Petermann, Laser diode modulation and noise, vol. 3 (Springer Science \& Business Media, 2012).

8. G. Giuliani, M. Norgia, S. Donati, and T. Bosch, J. Opt 4, S283 (2002).

9. R. Lang and K. Kobayashi, IEEE J. Quantum Electron. 16, 347 (1980).

10. E. Gagnon and J. F. Rivest, IEEE Trans. Instrum. Meas. 48, 693 (1999).

11. Y. L. Lim, M. Nikolic, K. Bertling, R. Kliese, and A. D. Rakić, Opt. Express 17, 5517 (2009).

12. S. Donati and M. Norgia, IEEE J. Sel. Top. Quantum Electron. 20, 104 (2014).

13. D. Guo and M. Wang, Appl. Opt. 46, 1486 (2007).

14. Z. Duan, Y. Yu, B. Gao, and C. Jiang, Opt. Commun. 389, 270 (2017).

15. S. Donati, G. Giuliani, and S. Merlo, IEEE J. Quantum Electron. 31, 113 (1995).

16. Y. L. Lim, K. Bertling, P. Rio, J. R. Tucker, and A. D. Rakic, Proc. SPIE 6038, 603810 (2006).

17. B. Liu, Y. Yu, J. Xi, Q. Guo, J. Tong, and R. A. Lewis, Appl. Opt. 56, 6962 (2017).

18. L. Xu, S. Zhang, Y. Tan, and L. Sun, Rev. Sci. Instrum. 85, 083111 (2014).

19. L. Scalise, Y. Yu, G. Giuliani, G. Plantier, and T. Bosch, IEEE Trans. Instrum. Meas. 53, 223 (2004).

20. L. Lv, H. Gui, T. Zhao, J. Xu, D. He, A. Wang, X. Chen, F. Li, H. Ming, and J. Xie, Proc. SPIE 5644, 829 (2005).

21. M. Norgia, A. Pesatori, and L. Rovati, IEEE Sens. J 12, 552 (2012).

22. J. Keeley, J. Freeman, K. Bertling, Y. L. Lim, R. A. Mohandas, T. Taimre, L. H. Li, A. D. R. Indjin, Dragan and, E. H. Linfield, A. G. Davies, and P. Dean, Sci. Rep. 7, 7236 (2017).

23. S. Zhang, Y. Tan, Z. Ren, Y. Zhang, and S. Zhang, Appl. Phys. B 116, 609 (2014).

24. R. C. Addy, A. W. Palmer, K. Thomas, and V. Grattan, J. Light. Technol. 14, 2672 (1996).

25. T. Yoshino, M. Nara, S. Mnatzakanian, B. S. Lee, and T. C. Strand, Appl. Opt. 26, 892 (1987).

26. D. Guo, M. Wang, and H. Hao, Appl. Opt. 54, 9320 (2015).

27. M. Wang and G. Lai, Rev. Sci. Instrum. 72, 3440 (2001).

28. N. Servagent, T. Bosch, and M. Lescure, IEEE J. Sel. Top. Quantum 6, 798 (2000).

29. N. Servagent, F. Gouaux, and T. Bosch, J. Opt. 29, 168 (1998).

30. C. Bes, G. Plantier, and T. Bosch, IEEE Trans Instrum Meas 55, 1101 (2006).

31. W. M. Wang, K. T. V. Grattan, A. W. Palmer, and W. J. O. Boyle, J. Light. Technol. 12, 1577 (1994).

32. C. Y. Chang, D. Choi, A. Locquet, M. J. Wishon, K. Merghem, A. Ramdane, F. Lelarge, A. Martinez, and D. S. Citrin, Appl. Phys. Lett. 108, 191109 (2016).

33. A. Mowla, B. W. Du, T. Taimre, K. Bertling, S. Wilson, and A. D. Rakić, Appl. Opt. 57, 4067 (2018).

34. R. Kliese, T. Taimre, A. A. A. Bakar, Y. L. Lim, K. Bertling, M. Nikolić, J. Perchoux, T. Bosch, and A. D. Rakić, Appl. Opt. 53, 3723 (2014).

35. Y. Yu, Y. Fan, and B. Liu, Proc. SPIE 10021, 10021U (2016).

36. C. Masoller and N. B. Abraham, Phys. Rev. A 57, 1313 (1998).

37. A. Locquet, B. Kim, D. Choi, N. Li, and D. S. Citrin, Phys. Rev. A 95, 023801 (2017).

38. D. D'Agostino, H. P. M. M. Ambrosius, M. K. Smit, and D. Lenstra, IEEE Photon. Technol. Lett. 27, 2292 (2015).

39. A. A. Sahai, B. Kim, D. Choi, A. Locquet, and D. S. Citrin, Opt. Lett. 39, 5630 (2014).

40. A. Ehtesham, U. Zabit, O. D. Bernal, G. Raja, and T. Bosch, IEEE Sensors J. 17, 7425 (2017).

41. Y. Fan, Y. Yu, J. Xi, and Q. Guo, IEEE J. Quantum Electron. 51, 1 (2015).

42. J. Perchoux, H. E. Dougan, F. Bony, and A. D. Rakic, Sensors, 2008 IEEE pp. 290-293 (2008).

43. S. Donati, IEEE J. Quantum Electron. 47, 1428 (2011). 


\section{FULL REFERENCES}

1. H. Hansen, K. Carneiro, H. Haitjema, and L. D. Chiffre, "Dimensional micro and nano metrology," CIRP Annals 55, 721 - 743 (2006).

2. A. J. Fleming, "A review of nanometer resolution position sensors: Operation and performance," Sens. Actuator A-Phys. 190, $106-126$ (2013).

3. S. Donati, "Developing self-mixing interferometry for instrumentation and measurements," Laser Photonics Rev. 6, $393-417$ (2012).

4. T. Taimre, M. Nikolić, K. Bertling, Y. L. Lim, T. Bosch, and A. D. Rakić, "Laser feedback interferometry: a tutorial on the self-mixing effect for coherent sensing," Adv. Opt. Photon. 7, 570-631 (2015).

5. D. J. Bell, T. J. Lu, N. A. Fleck, and S. M. Spearing, "Mems actuators and sensors: observations on their performance and selection for purpose," J. Micromechanics Microengineering 15, S153 (2005).

6. M. A. Haque and M. T. A. Saif, "A review of mems-based microscale and nanoscale tensile and bending testing," Exp. Mech 43, 248-255 (2003).

7. K. Petermann, Laser diode modulation and noise, vol. 3 (Springer Science \& Business Media, 2012).

8. G. Giuliani, M. Norgia, S. Donati, and T. Bosch, "Laser diode self-mixing technique for sensing applications," J. Opt 4, S283 (2002).

9. R. Lang and K. Kobayashi, "External optical feedback effects on semiconductor injection laser properties," IEEE J. Quantum Electron. 16, 347-355 (1980).

10. E. Gagnon and J. F. Rivest, "Laser range imaging using the self-mixing effect in a laser diode," IEEE Trans. Instrum. Meas. 48, 693-699 (1999).

11. Y. L. Lim, M. Nikolic, K. Bertling, R. Kliese, and A. D. Rakić, "Self-mixing imaging sensor using a monolithic vcsel array with parallel readout," Opt. Express 17, 5517-5525 (2009).

12. S. Donati and M. Norgia, "Self-mixing interferometry for biomedical signals sensing," IEEE J. Sel. Top. Quantum Electron. 20, 104-111 (2014).

13. D. Guo and M. Wang, "Self-mixing interferometry based on a double-modulation technique for absolute distance measurement," Appl. Opt. 46, 1486-1491 (2007).

14. Z. Duan, Y. Yu, B. Gao, and C. Jiang, "Absolute distance measurement based on multiple self-mixing interferometry," Opt. Commun. 389 , 270 - 274 (2017).

15. S. Donati, G. Giuliani, and S. Merlo, "Laser diode feedback interferometer for measurement of displacements without ambiguity," IEEE J. Quantum Electron. 31, 113-119 (1995).

16. Y. L. Lim, K. Bertling, P. Rio, J. R. Tucker, and A. D. Rakic, "Displacement and distance measurement using the change in junction voltage across a laser diode due to the self-mixing effect," Proc. SPIE 6038, 603810 (2006).

17. B. Liu, Y. Yu, J. Xi, Q. Guo, J. Tong, and R. A. Lewis, "Displacement sensing using the relaxation oscillation frequency of a laser diode with optical feedback," Appl. Opt. 56, 6962-6966 (2017).

18. L. Xu, S. Zhang, Y. Tan, and L. Sun, "Simultaneous measurement of refractive-index and thickness for optical materials by laser feedback interferometry," Rev. Sci. Instrum. 85, 083111 (2014).

19. L. Scalise, Y. Yu, G. Giuliani, G. Plantier, and T. Bosch, "Self-mixing laser diode velocimetry: application to vibration and velocity measurement," IEEE Trans. Instrum. Meas. 53, 223-232 (2004).

20. L. Lv, H. Gui, T. Zhao, J. Xu, D. He, A. Wang, X. Chen, F. Li, H. Ming, and J. Xie, "Theoretical and numerical analysis of polarization properties used as doppler velocimetry in vertical-cavity surface-emitting lasers," Proc. SPIE 5644, 829-835 (2005).

21. M. Norgia, A. Pesatori, and L. Rovati, "Self-Mixing Laser Doppler Spectra of Extracorporeal Blood Flow: A Theoretical and Experimental Study," IEEE Sens. J 12, 552-557 (2012).

22. J. Keeley, J. Freeman, K. Bertling, Y. L. Lim, R. A. Mohandas, T. Taimre, L. H. Li, A. D. R. Indjin, Dragan and, E. H. Linfield, A. G. Davies, and P. Dean, "Measurement of the emission spectrum of a semiconductor laser using laser-feedback interferometry," Sci. Rep. 7, 7236 (2017).

23. S. Zhang, Y. Tan, Z. Ren, Y. Zhang, and S. Zhang, "A microchip laser feedback interferometer with nanometer resolution and increased measurement speed based on phase meter," Appl. Phys. B 116, 609-616 (2014).

24. R. C. Addy, A. W. Palmer, K. Thomas, and V. Grattan, "Effects of external reflector alignment in sensing applications of optical feedback in laser diodes," J. Light. Technol. 14, 2672-2676 (1996).

25. T. Yoshino, M. Nara, S. Mnatzakanian, B. S. Lee, and T. C. Strand, "Laser diode feedback interferometer for stabilization and displacement measurements," Appl. Opt. 26, 892-897 (1987).

26. D. Guo, M. Wang, and H. Hao, "Displacement measurement using a laser feedback grating interferometer," Appl. Opt. 54, $9320-9325$ (2015).

27. M. Wang and G. Lai, "Displacement measurement based on fourier transform method with external laser cavity modulation," Rev. Sci. Instrum. 72, 3440-3445 (2001).

28. N. Servagent, T. Bosch, and M. Lescure, "Design of a phase-shifting optical feedback interferometer using an electrooptic modulator," IEEE J. Sel. Top. Quantum 6, 798-802 (2000).

29. N. Servagent, F. Gouaux, and T. Bosch, "Measurements of displacement using the self-mixing interference in a laser diode," J. Opt. 29, 168 (1998).

30. C. Bes, G. Plantier, and T. Bosch, "Displacement measurements using a self-mixing laser diode under moderate feedback," IEEE Trans Instrum Meas 55, 1101-1105 (2006).

31. W. M. Wang, K. T. V. Grattan, A. W. Palmer, and W. J. O. Boyle, "Self-mixing interference inside a single-mode diode laser for optical sensing applications," J. Light. Technol. 12, 1577-1587 (1994).

32. C. Y. Chang, D. Choi, A. Locquet, M. J. Wishon, K. Merghem, A. Ramdane, F. Lelarge, A. Martinez, and D. S. Citrin, "A multi-ghz chaotic optoelectronic oscillator based on laser terminal voltage," Appl. Phys. Lett. 108, 191109 (2016).

33. A. Mowla, B. W. Du, T. Taimre, K. Bertling, S. Wilson, and A. D. Rakić, "Polarization-sensitive laser feedback interferometry for specular reflection removal," Appl. Opt. 57, 4067-4074 (2018).

34. R. Kliese, T. Taimre, A. A. A. Bakar, Y. L. Lim, K. Bertling, M. Nikolić, J. Perchoux, T. Bosch, and A. D. Rakić, "Solving self-mixing equations for arbitrary feedback levels: a concise algorithm," Appl. Opt. 53, 3723-3736 (2014).

35. Y. Yu, Y. Fan, and B. Liu, "Self-mixing interferometry and its applications," Proc. SPIE 10021, 10021U (2016).

36. C. Masoller and N. B. Abraham, "Stability and dynamical properties of the coexisting attractors of an external-cavity semiconductor laser," Phys. Rev. A $\mathbf{5 7}$, 1313-1322 (1998).

37. A. Locquet, B. Kim, D. Choi, N. Li, and D. S. Citrin, "Initial-state dependence of the route to chaos of an external-cavity laser," Phys. Rev. A 95, 023801 (2017).

38. D. D’Agostino, H. P. M. M. Ambrosius, M. K. Smit, and D. Lenstra, "Integrated Laser With Optical Feedback Shows Suppressed Relaxation-Oscillation Dynamics," IEEE Photon. Technol. Lett. 27, 2292-2295 (2015).

39. A. A. Sahai, B. Kim, D. Choi, A. Locquet, and D. S. Citrin, "Mapping the nonlinear dynamics of a laser diode via its terminal voltage," Opt. Lett. 39, 5630-5633 (2014).

40. A. Ehtesham, U. Zabit, O. D. Bernal, G. Raja, and T. Bosch, "Analysis and implementation of a direct phase unwrapping method for displacement 
measurement using self-mixing interferometry," IEEE Sensors J. 17, 7425-7432 (2017).

41. Y. Fan, Y. Yu, J. Xi, and Q. Guo, "Stability limit of a semiconductor laser with optical feedback," IEEE J. Quantum Electron. 51, 1-9 (2015).

42. J. Perchoux, H. E. Dougan, F. Bony, and A. D. Rakic, "Photodiode-free doppler velocimeter based on self-mixing effect in commercial vcsels," Sensors, 2008 IEEE pp. 290-293 (2008).

43. S. Donati, "Responsivity and noise of self-mixing photodetection schemes," IEEE J. Quantum Electron. 47, 1428-1433 (2011). 